\title{
Delayed light emission to distinguish astrophysical neutrino flavors in IceCube
}

\section{The IceCube Collaboration}

http://icecube.wisc.edu/collaboration/authors/icrc17_icecube

E-mail: ansteuer@uni-mainz.de, lutz.koepke@uni-mainz.de

IceCube, a cubic kilometer Cherenkov detector, is an ideal instrument for the detection of astrophysical neutrino fluxes and rare interactions of exotic particles. One way to advance IceCube neutrino analyses would be the development of methods to identify the neutrino flavor and reaction type. While charged current interactions leading to long tracks of charged muons can be easily distinguished from those leading to particle cascades of $\mathscr{O}(10 \mathrm{~m})$ extension, the $17 \mathrm{~m}$ sensor distance makes it very challenging to identify the type of cascade as being due to the fragmentation of the struck nucleus or due to showers initiated by electrons or tauons. Only at very high energies, the average $\tau$ flight distance of $50 \mathrm{~m} / \mathrm{PeV}$, in principle, allows one to identify a $v_{\tau}$ interaction. So far, IceCube has not registered telltale events of such kind.

The creation of neutrons, spallation products and muons in hadronic showers offers an alternative handle to identify a hadronic interaction. However, the regular data acquisition system is restricted to a $\mathscr{O}(10 \mu \mathrm{s})$ readout window, limiting the detection to relativistic or low relativistic particles. Recently, the development of a spooling system to store the complete detector hit map for a configurable time interval made it possible to register a potential "afterglow" of highly energetic events. While the signal from Michel electrons from muon decay will be difficult to extract due to light scattering in the ice and afterpulses of the PMTs, the detection of $2.224 \mathrm{MeV}$ photons from neutron capture on hydrogen is promising due to the low PMT dark rates of the sensors in IceCube. One should note that luminescence in the ice could be a competing effect.

In this paper, we summarize the physics scope and particle identification opportunities, study the underlying physics of delayed signals and the expected detector response in a Cherenkov detector, discuss a newly installed automatic real time triggering system, with emphasis on the the capabilities and limitations of the data acquisition system for such an analysis, and outline the expected potential of IceCube for particle identification through the use of delayed signals.

Corresponding author: A. Steuer* and L. Köpke

Johannes Gutenberg University Mainz, Germany

35th International Cosmic Ray Conference - ICRC2017

10-20 July, 2017

Bexco, Busan, Korea

\footnotetext{
*Speaker.
} 


\section{Delayed light emission processes and physics scope of cascade identification}

The striking background reduction by the coincident registration of a delayed neutron signal was vital for the first detection of $\bar{v}_{\mathrm{e}}$ in the 1956 neutrino discovery experiment by Cowan and Reines. Since then, many experiments have been relying on characteristic delayed signals to identify rare neutrino interactions in the presence of large backgrounds. The possible use of delayed signals in IceCube [1] for flavor-identification was pointed out in a recent paper by S. Li, M. Bustamante and J.F. Beacom [2], which stimulated the ongoing efforts in IceCube. The detection of Michel electrons from muon decay is challenging due to light scattering in the ice and the occurrence of afterpulses. Spallation products, such as those from the decay of ${ }^{16} \mathrm{~N}$ or ${ }^{12} \mathrm{~B}$ nuclei, contribute less than $1 \%$ to delayed signals. In this note, we therefore concentrate on the detection of the "neutron echo". Before being captured, the neutrons are moderated by elastic scattering and the number of neutrons $d N$ captured in a time interval $d t$ is given by $d N \propto-N \sigma v d t \propto-N \lambda d t$, with $\sigma \propto 1 / v$. Hence, one observes an exponential time dependence of the capture process. In ice, the effective moderation time constant $\tau_{\mathrm{m}}=1 / \lambda$ amounts to $\tau_{\mathrm{m}} \approx 190 \mu \mathrm{s}$, with a spread of $\approx 18 \mu \mathrm{s}$ from hadronic shower fluctuations. Signals from neutron capture were measured by Super-Kamiokande in water, both with a neutron source and cosmic ray muons $[3,4]$ and confirm these simulation results.

When deciphering the origin of astrophysical neutrinos, their flavor composition plays a similarly important role as their direction and energy dependence. A flavor composition at the source of $\left(\frac{v_{\mathrm{e}}}{v_{\text {total }}}: \frac{v_{\mu}}{v_{\text {total }}}: \frac{v_{\tau}}{v_{\text {total }}}\right)=\left(\frac{1}{3}: \frac{2}{3}: 0\right)$, expected for pion decay, is transformed by incoherent neutrino vacuum mixing to approximately $\left(\frac{1}{3}: \frac{1}{3}: \frac{1}{3}\right)$ at Earth, providing a large fraction of $\tau$ neutrinos through oscillation. As can be seen from Fig. 1 (left), present data have little power to separate $v_{\mathrm{e}}$ from $v_{\tau}$ initiated showers. Methods to experimentally separate these contributions are therefore needed. In IceCube, a positive $v_{\tau}$ identification has not yet occurred (see e.g. [5, 6]). A further flavor
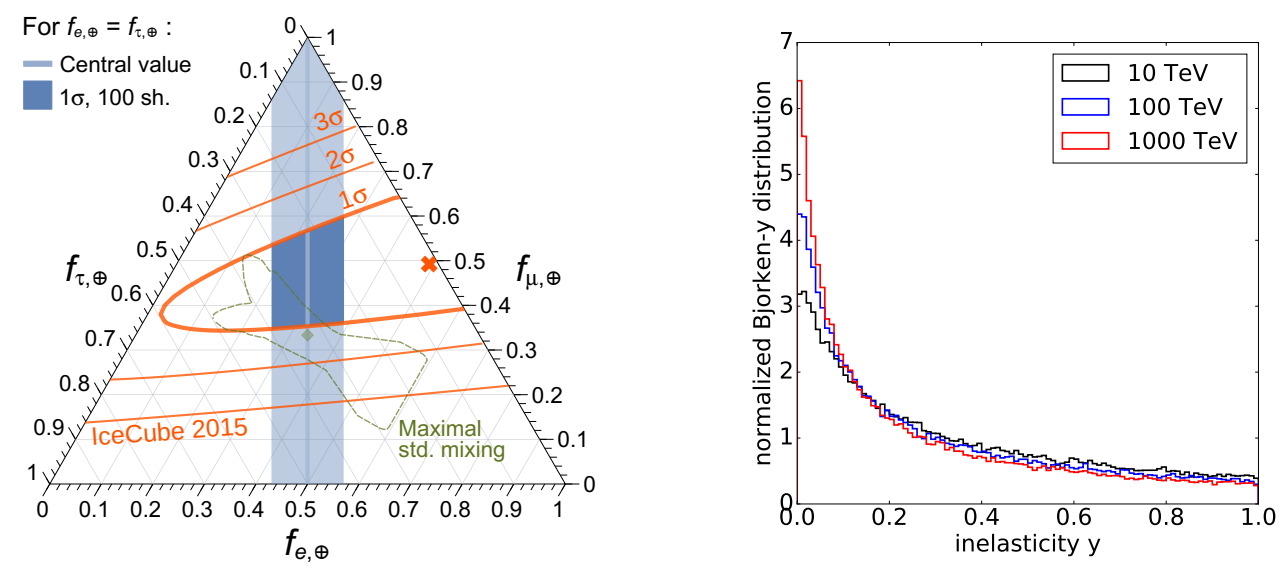

Figure 1: Left: Flavor composition $f_{l}(l=e, \mu, \tau)$ of astrophysical neutrinos at Earth. Read each axis parallel to its respective ticks. Orange: IceCube fit [7], blue: prediction by [2] assuming 100 showers of $100 \mathrm{TeV}$ under ideal conditions for delayed photon observation, green: allowed range by current mixing uncertainties. Right: normalized $y$-distributions for $E_{V}=10,100$ and $1000 \mathrm{TeV}$ using the parametrization of [8].

sensitive category of events are $\bar{v}_{\mathrm{e}}$ reacting on $\mathrm{e}^{-}$in the electronic clouds of ice molecules, thereby creating on-shell $\mathrm{W}^{-}$bosons. The resonant reaction leads to a peak in the neutrino cross section at around $6.3 \mathrm{PeV}$ ("Glashow resonance"). IceCube should be on the verge of detecting such events 
if the spectrum is not cut off at high energies. As the $\mathrm{W}^{-}$decays to hadrons $69.8 \%$ of the time, including hadronic $\tau$ decays, their identification as being hadronic would sharpen the interpretation as being due to the Glashow resonance.

A neutrino interacts with a nucleon $N$ via the charged current reaction $v_{l}+N \rightarrow l+X$ or the neutral-current channel $v_{l}+N \rightarrow v_{l}+X$, where $l=\mathrm{e}, \mu$ or $\tau$ and $X$ represents hadrons produced in the fragmentation of the target nuclei. The distribution of the energy fraction $y$ ("inelasticity") carried by final-state hadrons peaks at $y=0$ with a long tail towards higher $y$-values; the average decreases with energy, yielding e.g. $\langle y\rangle \approx 0.3$ for $100 \mathrm{TeV} v$ and $\bar{v}$ interactions (see Fig. 1 right).

For a given reconstructed event energy in the detector, the contribution of neutral current interactions of all flavors depends on the assumed spectral index and on the $v$ energy, as the inelasticity distribution is peaked at low values and the neutrino spectrum is steeply falling ( $\approx 7 \%$ neutral current contribution for $\gamma=2.6$ and $100 \mathrm{TeV}$ detected energy). An excess of high energy hadronic showers would therefore be an intriguing indication of unexpected physics.

Long tracks are produced by $v_{\mu}$ charged current interactions and, in $17 \%$ of the cases, in $\tau \rightarrow \mu v_{\mu} v_{\tau}$ decays. Approximately $65 \%$ of the $\tau$ leptons decay hadronically with $\approx 25 \%$ of the energy escaping undetected by neutrinos. In the case of charged current $v_{\mathrm{e}}$ interactions, electronand hadron initiated showers combine approximately to the total neutrino energy with fractions determined by the inelasticity distribution.

There are many possibilities to explore beyond the Standard Model physics if one could distinguish between hadronic, tauonic and electromagnetic showers. As can be seen from Fig. 1 (left), the allowed range for standard neutrino mixing for different production scenarios is rather restricted. Therefore the flavor composition analysis could be an interesting indicator of new physics. Similarly, neutrino cross sections above $1 \mathrm{TeV}$ have never been probed experimentally.

The IceCube excess of neutrinos exceeding $100 \mathrm{TeV}$ energy has led to much speculation with regards to their origin, including top down scenarios. For example, it has been argued that the observed spectrum is indicative for the interaction of boosted dark matter, where a very heavy dark matter particle decays and thereby produces a strongly boosted secondary particle that triggers a nuclear recoil in the IceCube detector $[9,10]$. As such a recoil would be completely hadronic in nature and the natural occurrence of neutral current interactions is rare, such a scenario could be immediately tested if the shower-type information were available.

\section{Shower development, physics of delayed signals and detector response}

Electromagnetic showers start out with electrons, positrons and gamma rays and only few pions and nucleons are subsequently produced by photo-nuclear processes. A hadronic shower starts out with mesons and nucleons, building up a progressively larger fraction of electromagnetic particles from prompt $\pi^{0} \rightarrow \gamma \gamma$ decays. The shower development ends once energy-loss processes dominate the particle production. At this stage, pions contribute $\approx 10 \%$ to the Cherenkov light in hadronic showers and less than $1 \%$ in electromagnetic showers. Evaporated neutrons are characteristic of hadronic showers and are mostly absent in electromagnetic showers. Neutrons with $\mathrm{MeV}$ energies thermalize by undergoing a random walk until they are captured on hydrogen forming an excited deuteron that subsequently decays with the emission of a $2.224 \mathrm{MeV}$ photon. At the end of the shower development, $\pi^{-}$are typically captured by nuclei after coming to rest by 
ionization. $\pi^{+}$decay to $\mu$ 's which subsequently, after decaying with an average lifetime of $2.2 \mu \mathrm{s}$, produce positrons with $\approx 35 \mathrm{MeV}$ energy. Note that the Cherenkov yields from hadronic and electromagnetic interactions are not identical but close at high energies, as the electromagnetic fraction in hadronic showers rises from $\approx 75 \%$ at $1 \mathrm{TeV}$ to $\approx 92 \%$ at $1 \mathrm{PeV}$. As a consequence, the relative number of Cherenkov photons seen in a hadronic shower compared to a purely electromagnetic shower reaches $94 \%$ at $1 \mathrm{PeV}$ energy (see Fig. 2).

\subsection{Pythia}

\section{and Geant4 simulation without detector response}

The deep inelastic scattering of $v$ 's of all species on protons has been simulated in Pythia 8 including the DIRE-plug-in [12] to implement all order radiative corrections using a dipole re-summation technique. All final state particles with their kinematic information

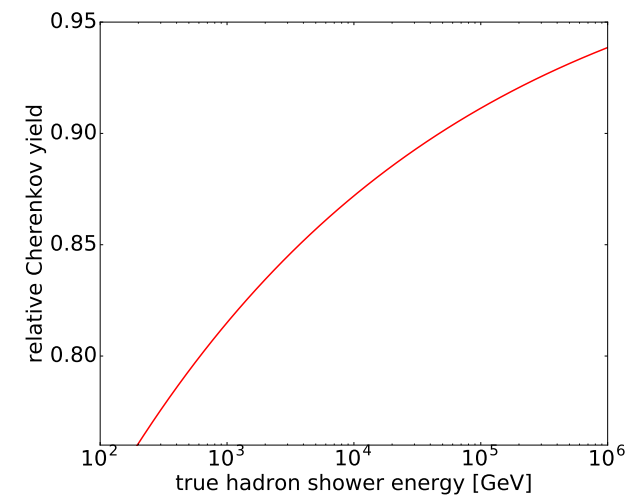

Figure 2: Cherenkov photon yield from hadronic showers relative to electromagnetic showers (Pythia particle spectra plus Geant4 hadronic showers [11]). are then written to files that can be read by Geant 4 and the standard IceCube simulation. Geant 4 is then used in CPU-intensive simulations of particles in natural ice ${ }^{1}$. The number of delayed photons due to neutron capture and the total number of Cherenkov photons are stored for each particle type and energy. Parametrized results are then provided to a fast simulation. The sensitivity to Geant4's hadronic models (physics lists) and cuts has been investigated and a comparison to a FLUKA calculation [2] has been performed. The predicted strength of the neutron capture signal varies by $\approx 40 \%$, however, the uncertainty may be even larger [2].

The result of the pure Geant 4 simulation, which disregards detector effects and thus represents the optimal resolution limited only by the shower development, is shown in Fig. 3 (left) as the ratio of the number of neutron capture induced Cherenkov photons over the total photon count. There is a clear separation of shower types seen: charge current $v_{\mathrm{e}}$ interactions follow the inelasticity distribution in shape and neutral current interactions produce a symmetric Gaussian-like distribution, with on average 0.00055 delayed per prompt photon. The $25 \%$ relative spread reflects the fluctuations in the hadron shower. The low $\tau$ decay multiplicity and $\approx 25 \%$ undetected energy in hadronic $\tau$ decays from neutrinos lead to a shift and broadening of the distribution.

In order to quantify the optimal flavor discrimination potential when being limited only by physical fluctuations, we follow Ref. [2] in defining a Bayesian probability that takes into account a hypothesis on the initial flavor ratio and the rareness of neutral current decays. A Bayesian approach is useful in the view of combining particle identification measures and to visualize systematic dependencies. Figure 3 (right) shows the expected reaction discrimination potential for the assumption of an equal partition of flavors between astrophysical neutrinos. The discrimination improves slightly with rising energy due to the tightening of the inelasticity distribution (not shown). The band indicates the dependence on the assumption of the spectral index. Clearly, the distribution

\footnotetext{
${ }^{1}$ The ice is assumed to be made up of $89.7 \%{ }^{1} \mathrm{H}_{2}^{16} \mathrm{O}, 0.2 \%{ }^{1} \mathrm{H}_{2}^{18} \mathrm{O}_{2}, 0.03 \%{ }^{1} \mathrm{H}^{2} \mathrm{H}^{16} \mathrm{O}$ as well as $\approx 10 \%$ of natural air, whith contributions given in volume percent.
} 

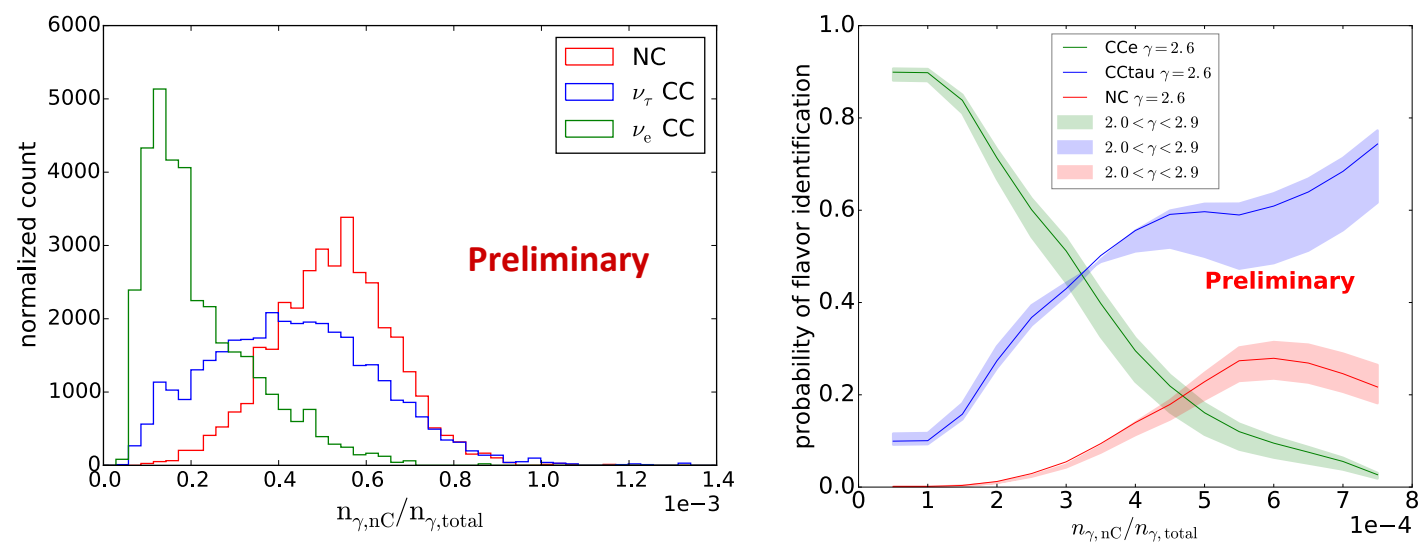

Figure 3: Left: Geant4 result on the ratio of neutron capture induced and total Cherenkov photons. Right: Bayesian shower identification probability according to [2] (Appendix A), assuming neutrino flavor equipartition, $100 \mathrm{TeV}$ detected energy and a range in spectral indexes.

is sensitive to the flux assumption and would look very different when e.g. assuming the expected ratio of atmospheric neutrinos or prompt neutrinos from charm. In order to determine a possible "new physics" contribution, we will choose a frequentist approach and compare the data to the null hypothesis of a standard event decompositon.

\subsection{IceCube simulation}

The events generated by Pythia and DIRE were interfaced with the Geant 4 based version of the IceCube Monte Carlo and processed to detection level, including a likelihood based energy reconstruction [13] for cascades. Because the CPU time rises strongly with energy, we restricted ourselves to neutrino interactions with reconstructed energies between 100-150 TeV. In Fig. 4 we show the IceCube per event response to delayed photons in hadron showers. In this particular simulation, the detector noise simulation had been switched off for clarity. While one clearly observes the fall-off due to delayed photons from neutron capture, there is a observable gap between 10-45 $\mu$ s separating "prompt" and "delayed" photons. This behavior is due to deadtime effects in the digitizing electronics which will be described in the following paragraph in detail.

\subsection{Deadtime effects of the electronics}

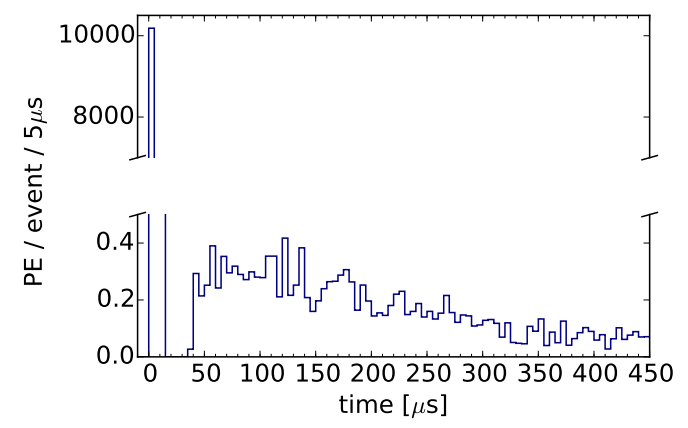

Figure 4: IceCube simulation of neutral current events with registered energies between 100$150 \mathrm{TeV}$ (detected photo electrons (PE) for 5 optical modules with highest signal expectation).

In IceCube, Cherenkov photons emitted from charged particles are detected by $25.4 \mathrm{~cm}$ diameter photomultipliers which amplify the charge of the recorded PE by roughly a factor of $10^{7}$. These signals are stored by tandem ATWDs with $300 \mathrm{MHz}$ sampling rate for $\approx 427 \mathrm{~ns}$ and are digitized and transmitted afterwards [1]. Each ATWD has 3 input channels with factors of 16, 2, and 0.25 amplification in order to increase the 10 bit resolution to effectively 15 bits. An FADC with 10 bit 
resolution and $40 \mathrm{MHz}$ sampling rate can record up to $6.4 \mu \mathrm{s}$. The combination of ATWDs and an FADC allows for a nearly deadtime free recording of prompt particle interactions including delays that photons suffer from scattering in the ice. However, when extending the readout to much larger time spans, several deadtime effects start impacting the data taking due to the digitization process ${ }^{2}$. Obviously, the deadtime hinges on the number of amplification channels used and therefore strongly depends on the shower position and energy (see Fig. 5 right). Often, afterpulses start the second ATWD so that the deadtime can occur after some delay and introduces a complex repetitive pattern.

In order to acquire the necessary statistics to smoothen the deadtime probability density distribution for the later use in a likelihood fit, a fast code was implemented to simulate the photon propagation, afterpulses and the electronics response. Figure 5 shows an example of the deadtime effect in the five optical modules with the highest signal expectation as function of time. This result for a $750 \mathrm{TeV}$ shower was obtained from the high statistics simulation discussed above.
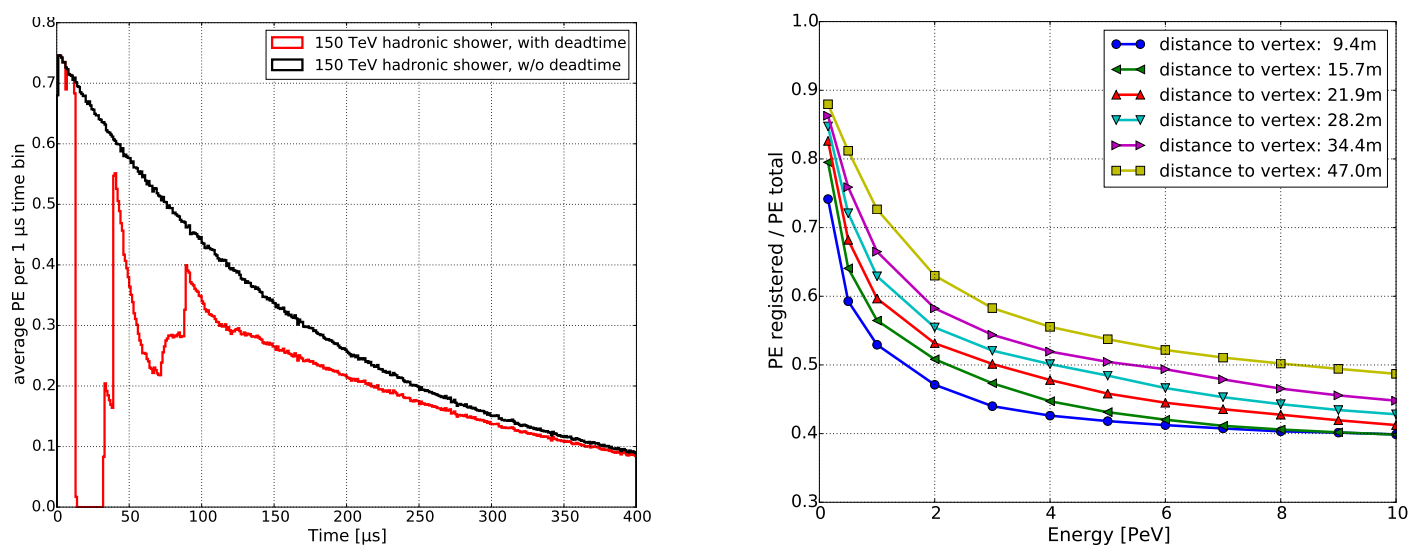

Figure 5: Left: example for the effect of the deadtime on an event with $150 \mathrm{TeV}$ shower energy averaged over five optical modules with distances to the shower vertex between $15.4 \mathrm{~m}$ and $40.3 \mathrm{~m}$. Right: overall loss of registered PEs due to deadtime as function of energy and shower vertex distance to the closest optical module.

\section{High energy starting events, real time filtering and HitSpool data acquisition}

In 2013, IceCube reported the discovery of an astrophysical flux of neutrinos in the $60 \mathrm{TeV}$ to multiple PeV energy range [14]. The key to this discovery was a focus on events in which the neutrino vertex is contained in a fiducial volume that covers slightly less than half of IceCube. The outer surface of the detector is used to reject events with the signature of atmospheric $\mu$ 's and $v$ 's and a requirement of at least 1500 detected PEs is imposed. Below 6000 PEs, $95 \%$ of the events are due to atmospheric muons that are nor tagged by the veto layers. The high energy starting event analysis therefore concentrates on events with $>6000$ PEs, roughly equivalent to a minimal energy of $30 \mathrm{TeV}$, with an estimated background of atmospheric neutrinos and muons of roughly $17 \%$ and $23 \%$, respectively [15]. Cascades signatures dominate the sample.

\footnotetext{
${ }^{2}$ The recording on ATWDs and FADC is blocked by $\Delta t=t_{\text {reset }}+t_{\mathrm{DAQ}}+t_{\text {clear }} \cdot(4-n)+t_{\text {readout }} \cdot n<88.6 \mu \mathrm{s}$, with $n \in\{1,2,3\}$ being the number of ATWD input channels involved. Reset, data taking, clearing and readout times are given by $t_{\text {reset }}=225 \mathrm{~ns}, t_{\mathrm{DAQ}}=426.67 \mathrm{~ns}, t_{\text {clear }}=950 \mathrm{~ns}$, and $t_{\text {readout }}=29 \mathrm{~ns}$.
} 
The high energy starting event sample is well understood, is automatically selected by a filter script at South Pole with a median latency of $\approx 40 \mathrm{~s}$, is of sufficiently small size and thus very well suited for the analysis described in this note. In order to provide access to all hits independent of the time of a trigger, the hit stream from each optical module is buffered in a

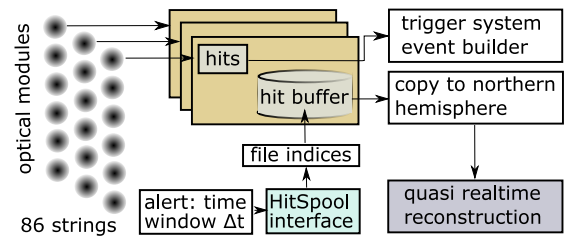

Figure 6: Data flow for saving the detector hit image (HitSpooling) for $\Delta t=1 \mathrm{~s}$. dedicated industrial computer, where the hits are chronologically ordered and packaged. A copy of the raw data is written (see Fig. 6) to a circular buffer on disk (HitSpool [1]). In the case of a high energy starting event candidate with $>1500$ registered photoelectrons (PE), a request is sent to the HitSpool system to store hits within $1 \mathrm{~s}$ of the trigger and to initiate an automatic transfer of data to the North and the subsequent processing. The system has been working reliably since February 2016. The automatic processing models the triggers, removes backgrounds, reconstructs energies and provides various file formats to the user. As of April 2017, 1270 events have been registered, with around 20 events in a blinded region above $6000 \mathrm{PE}$. Analyzing the five optical modules with the highest photon count expectation and subtracting the average estimated dark count background $(\approx 0.0006$ hits per $\mu \mathrm{s})$, the distribution in Fig. 7 shows clear evidence for delayed hits for $t>50 \mu \mathrm{s}$. In fact, the signal is surprisingly large, given the dominance of atmospheric muons (95\%) in the sample. An explanation may be that the background is dominated by catastrophic energy losses, $\approx$ $15 \%$ of which have a photonuclear origin. A quantitative study is on its way.

In order to optimally extract the signal in the presence of dark noise, one can go one step further and use a likelihood approach. The likelihood

$$
L\left(n_{s}\right)=\frac{\exp \left(-\mu_{b}\right) \cdot \mu_{b}}{\Gamma\left(N-n_{s}+1\right)} \prod_{j=1}^{N_{\mathrm{HIT}}} \prod_{i=1}^{N_{\mathrm{DOM}}}\left(\frac{n_{s}}{N} \cdot P_{s(i, j)}(\vec{r}, t)+\frac{N-n_{s}}{N} \cdot P_{b}\right)
$$

exploits the expected temporal distribution and the expected position dependent energy deposition of the signal.

In equation $3.1, n_{s}$ denotes the number of delayed photons, $N$ is the number of all registered photons, $\mu_{b} \approx$ $0.55 \cdot N_{\mathrm{DOM}}$ is the expected number of dark counts and $N_{\mathrm{DOM}}$ is the number of optical modules taken into account in the fit with $N_{\text {HIT }}$ hits per optical module. The signal probability $P_{s(i, j)}(\vec{r}, t)=P_{s(i, j)}(\vec{r}) \cdot P_{s(i, j)}(t)$ can be factorized in a spacial part $P_{s(i, j)}(\vec{r})=q_{i} /\left(\sum_{i=1}^{N_{\text {DOM }}} q_{i}\right)$ and a temporal part $P_{s(i, j)}\left(t_{j}\right)=\frac{f_{\text {dt }}}{\tau} \exp \left(-t_{j} / \tau\right)$, with the expected number of photons per DOM $q_{i}$, the neutron moderation time $\tau=190 \mu$ s and the time and po-

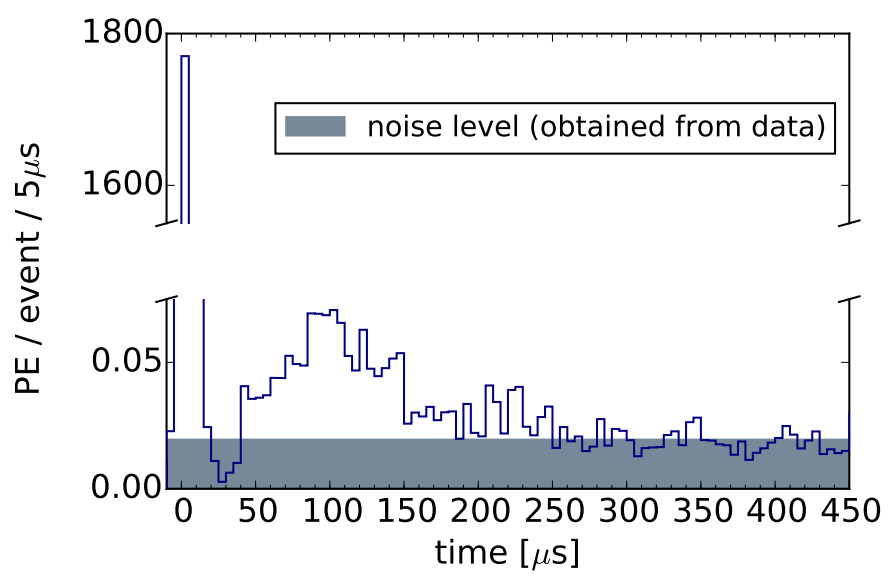

Figure 7: Stacked time distribution for 1250 high energy starting events with 1500-6000 PE recorded. 
for an analysis period $\Delta t \approx 1 \mathrm{~ms}$. A toy Monte Carlo study showed that deposited hadronic energies larger than $\approx 100 \mathrm{TeV}$ are required to obtain a significant result on the fraction of delayed photons and that statistical uncertainties better than $25 \%$ will be achieved if the hadronic energy exceeds $500 \mathrm{TeV}$. Including more than $N_{\mathrm{DOM}}=15$ optical modules in the likelihood fit will not significantly improve the results.

\section{Summary}

Delayed signals in neutrino reactions may be used to classify electromagnetic, hadronic and tau induces showers and thus provide a potential tool to determine the neutrino flavor composition and to test the data for unexpected phenomena. Preliminary Monte Carlo studies of the IceCube response to delayed photons from neutron capture indicate that the method becomes sensitive if hadronic energies exceed $\approx 100 \mathrm{TeV}$. A search in an atmospheric muon dominated, real-time extracted data set shows clear evidence for such delayed processes. If alternative sources [16] can be excluded and if large uncertainties in the simulation of hadronic processes can be reduced [17] and dead time effects are properly handled, the method will provide a complimentary flavor discrimination tool in the future.

\section{References}

[1] IceCube Collaboration, M. G. Aartsen et al., JINST 12 (2017) P03012.

[2] S. W. Li, M. Bustamante and J. F. Beacom, [astro-ph.HE/1606.06290].

[3] Super-Kamiokande Collaboration, H. Watanabe et al., Astropart. Phys. 31 (2009) 320.

[4] Super-Kamiokande Collaboration, H. Zhang et al., Astropart. Phys. 60 (2015) 41.

[5] IceCube Collaboration, M. G. Aartsen et al., Phys. Rev. D 93, no. 2, 022001 (2016).

[6] IceCube Collaboration, PoS ( ICRC2017) 974 (these proceedings).

[7] IceCube Collaboration, M. G. Aartsen et al., Phys. Rev. Lett. 114 (2015) 171102.

[8] A. Connolly, R. S. Thorne and D. Waters, Phys. Rev. D83 (2011) 113009.

[9] J. Kopp, J. Liu and X.P. Wang, JHEP04 (2015) 105.

[10] A. Bhattacharya, R. Gandhi, A. Gupta and S. Mukhopadhyay, JCAP 05 (2017) 002.

[11] L. Rädel, Master's thesis, RWTH Aachen (2012).

[12] S. Höche and S. Prestel, Eur. Phys. J. C75 (2015) 461.

[13] IceCube Collaboration, M. G. Aartsen et al., JINST 9 (2014) P03009.

[14] IceCube Collaboration, M. G. Aartsen et al., Science 342 (2013) 1242856.

[15] IceCube Collaboration, PoS ( ICRC2015) 1081 (2016).

[16] IceCube Collaboration, PoS ( ICRC2017) 1060 (these proceedings).

[17] I. Anghel et al., ANNIE LOI, [physics.ins-det/1504.01480]. 